\title{
Zeitgerechte Diagnosestellung bei Menschen mit Demenz: der Bayerische Demenz Survey (BayDem)
}

\section{Timely Diagnosis of Dementia: Results of the Bavarian Dementia Survey (BayDem)}

\section{(ㄷ) (1) $(8) \Theta$}

\author{
Autoren \\ Franziska Wolff', Nikolas Dietzel' ${ }^{1}$, Linda Karrer¹, André Kratzer², Manuela Hess', Elmar Gräßel², Peter Kolominsky- \\ Rabas $^{1}$
}

Institute

1 Interdisziplinäres Zentrum für Health Technology Assessment (HTA) und Public Health (IZPH), FriedrichAlexander-Universität Erlangen-Nürnberg (FAU), Erlangen

2 Zentrum für Medizinische Versorgungsforschung, Psychiatrische und Psychotherapeutische Klinik, Universitätsklinikum Erlangen, Friedrich-AlexanderUniversität Erlangen-Nürnberg (FAU), Erlangen

\section{Schlüsselwörter}

Demenz, zeitgerechte Diagnose, Dauer bis zur Diagnose, verzögerte Diagnose

\section{Key words}

dementia, timely diagnosis, time to diagnosis, diagnostic delay

\section{Bibliografie}

DOI https://doi.org/10.1055/a-1031-9559

Online-Publikation: 20.12.2019

Gesundheitswesen 2020; 82: 23-29

(c) Georg Thieme Verlag KG Stuttgart · New York

ISSN 0941-3790

\section{Korrespondenzadresse}

Univ.-Prof. Dr. med. Peter Kolominsky-Rabas, MBA

Interdisziplinäres Zentrum für Health Technology

Assessment (HTA) und Public Health (IZPH)

Friedrich-Alexander-Universität Erlangen-Nürnberg (FAU)

Schwabachanlage 6

91054 Erlangen

peter.kolominsky-rabas@fau.de

\section{ZUSAMMENFASSUNG}

Hintergrund Demenzdiagnosen werden häufig erst bei fortgeschrittener Symptomatik gestellt. Die Sicherstellung einer zeitgerechten Diagnose stellt jedoch die Grundlage für die Inanspruchnahme von Therapie- und Unterstützungsleistungen für Menschen mit Demenz (MmD) sowie für deren pflegende Angehörige dar. Bisher haben nur wenige internationale Stu- dien Einflussfaktoren auf die Dauer von den ersten subjektiv wahrgenommenen Symptomen bis zur Diagnosestellung analysiert. Ziel dieser Analyse ist es daher, die Zeitspanne von den ersten wahrgenommenen Symptomen aus der Perspektive der unterstützenden Angehörigen bis zur Erstdiagnose einer Demenz zu erfassen sowie potenzielle soziodemografische Prädiktoren für eine zeitgerechte Diagnose zu identifizieren.

Methode Der Bayerische Demenz Survey (BayDem) ist eine multizentrische Längsschnittstudie, die in 3 Regionen (Dachau, Erlangen, Kronach) in Bayern durchgeführt wurde. Projektteilnehmer/innen waren Menschen mit Demenz (MmD) nach ICD10, sowie deren pflegende Angehörige. Die Verlaufsdaten wurden in standardisierten, persönlichen Interviews in enger Zusammenarbeit mit lokalen Akteuren vor Ort erhoben. Zur Analyse von potenziellen soziodemografischen Prädiktoren einer zeitgerechten Diagnosestellung innerhalb von 9 Monaten nach Erkennung der ersten Symptome wurden binäre-logistische Regressionen durchgeführt.

Ergebnisse Die mediane Dauer von der Wahrnehmung der ersten Symptome bis zur Diagnosestellung betrug bei den Teilnehmenden 16 Monate. Neben dem Alter (zum Zeitpunkt der ersten wahrgenommenen Symptome), ist die Schulbildung von männlichen MmD mit einer zeitgerechten Diagnosestellung innerhalb von 9 Monaten nach Wahrnehmung der ersten Demenzsymptome assoziiert. Es konnte keine Assoziation mit dem Wohnort oder der Wohnsituation belegt werden.

Schlussfolgerungen Es zeigte sich, dass die Diagnosestellung häufig mit deutlicher Verzögerung erfolgt. Deshalb erscheint es notwendig, dass im primärärztlichen Bereich strukturierte Zugangswege zu einer „zeitgerechten Diagnostik“ weiterentwickelt werden.

\section{ABSTRACT}

Background In many cases, people with dementia (PWD) receive their first diagnosis at an advanced stage of the disease. A timely diagnosis, however, is crucial for the utilization of therapies and support services for PWD and their caregivers. So far, only a few international studies have analysed predictors of the time lapse between the first perceived symptoms and diagnosis. The aim of this study was to assess the time span 
from the first symptoms of dementia until the first dementia diagnosis and to identify predictors of a timely diagnosis.

Methods The Bavarian Dementia Survey (BayDem) is a multicentre, longitudinal study at 3 different sites in Bavaria, Germany. Participants were PWD as defined by ICD-10 and their informal caregivers. Data was collected by standardized faceto-face interviews in cooperation with local actors. For the analysis of potential sociodemographic predictors of a timely diagnosis within 9 months after the perception of the first symptoms, binary logistic regressions were used.
Results The median length from the perception of the first symptoms until diagnosis was 16 months. Predictors of a timely diagnosis for male PWD were age at the time of the first perceived symptoms and education. There was no association between a timely diagnosis and the place of residence or living situation of the PWD.

Conclusions Diagnosis of dementia is often made with a huge delay. Therefore, it is necessary to enhance structured access routes to a timely diagnosis in primary care.

\section{Einleitung}

\section{Hintergrund - Demenzerkennung in der primärärztlichen Versorgung}

Die Anzahl der derzeit in Deutschland lebenden Menschen mit Demenz (MmD) wird auf knapp 1,7 Mio. geschätzt [1]. Eine formelle Demenzdiagnose erfolgt jedoch auch in Industrienationen häufig erst im fortgeschritten Stadium [2]. Eine bevölkerungsbasierte Studie in Mecklenburg-Vorpommern zeigt, dass im primärärztlichen Bereich lediglich bei $40 \%$ der positiv auf Demenz gescreenten Personen eine formale Demenzdiagnose dokumentiert wurde [3]. Die German Study on Ageing Cognition and Dementia (AgeCoDE-Studie) gibt zudem Hinweise darauf, dass die Beurteilung der kognitiven Leistungsfähigkeit von älteren Patienten durch Hausärzte/ innen neben der objektiven kognitiven Testleistung auch durch weitere unabhängige Faktoren beeinflusst wird. Demnach werden ältere Patienten mit eingeschränkter Mobilität, Multimorbidität oder Hörproblemen häufiger als kognitiv beeinträchtigt eingestuft. Es zeigte sich zudem, dass kognitive Beeinträchtigungen von Patienten, die dem Hausarzt/in hingegen sehr gut bekannt sind, vermehrt unerkannt blieben [4]. Dies gilt auch für MmD die alleine leben [5]. Barrieren für eine frühzeitige Diagnosestellung in der Primärversorgung auf Ebene der Gesundheitsanbieter können gemäß internationalen Studien bspw. auch auf diagnostischen Unsicherheiten zu Beginn des Demenzsyndroms, fehlender Kommunikationskompetenz oder der Priorisierung von somatischen vor psychischen $\mathrm{Er}$ krankungen beruhen. Auch patientenseitig können Faktoren wie Krankheitsnegierung, Besorgnis vor dem Verlust von Fähigkeiten und potenzieller Stigmatisierung zu Verzögerungen in der Demenzerkennung führen $[6,7]$.

\section{„Zeitgerechte“ Diagnostik}

Die europäische Demenz-Initiative ALzheimer's COoperative Valuation in Europe (ALCOVE) empfiehlt, dass eine „zeitgerechte“ Demenzdiagnose zum Zeitpunkt der ersten Wahrnehmung kognitiver Veränderungen durch den MmD oder dessen pflegenden Angehörigen erfolgen sollte. Eine „zeitgerechte“ Diagnostik wird einer flächendeckenden „frühen“ Diagnostik in der präklinischen Phase aufgrund des noch fehlenden Nutzens für den Patienten vorgezogen. Der Zugang zu einer „zeitgerechten“ Demenzabklärung bildet gemäß ALCOVE Empfehlung die Grundlage für selbstbestimmte Entscheidungen über die Versorgungsplanung von MmD [8]. Weitreichende Evidenz besteht zudem darüber, dass eine zeitgerechte
Demenzerkennung die Voraussetzung für eine evidenzbasierte Behandlung, Beratung und Unterstützung von MmD und deren Angehörigen darstellt $[2,7]$.

Eine internationale Metanalyse untersuchte Präferenzen für oder gegen eine Demenzdiagnosemitteilung von Personen mit bestehenden kognitiven Einschränkungen sowie von Personen ohne kognitive Beeinträchtigungen. Es zeigte sich, dass der Wunsch nach Autonomieerhaltung der Hauptgrund dafür ist, dass sowohl die überwiegende Mehrheit der Personen mit kognitiven Beeinträchtigungen (84,8\%) als auch Personen ohne bestehende kognitive Beeinträchtigungen (90,7\%) im Falle der Entwicklung eines Demenzsyndroms eine Diagnosemitteilung präferieren [9]. Ferner verdeutlicht eine repräsentative deutsche Studie, dass auch die überwiegende Mehrheit der Erwachsenenbevölkerung (87,9\%) den Erhalt einer frühen Demenzdiagnose favorisiert [10].

\section{Einflussfaktoren auf eine zeitgerechte Diagnostik}

Die Autoren einer australischen, postalischen Studie [11] identifizierten 2 Bereiche in denen Verzögerungen im Patientenpfad auftreten können. Zum einen in der Zeitspanne von der Wahrnehmung erster Symptome bis zur ersten Kontaktaufnahme mit einem Gesundheitsanbieter zur Abklärung der Symptomatik, zum anderen der Zeitraum von der ersten Kontaktaufnahme bis zur gesicherten Diagnosestellung. Die durchschnittliche Gesamtzeitspanne belief sich auf 3,1 Jahre [11]. Ebenso einen Zeitraum von 3 Jahren ermittelte eine britische Studie, die in persönlichen Interviews den Patientenpfad von den ersten identifizierten Symptomen bis zum Zeitpunkt des Erreichens einer Gedächtnisambulanz erhob [12].

Feldman et al. [13] konnten zeigen, dass britische MmD, die innerhalb von 3 Monaten nach der Wahrnehmung erster Symptome Hilfe aufsuchten, durchschnittlich 16 Monate bis zur Diagnosestellung benötigten. Hingegen erhielten MmD, die erst später Hilfe in Anspruch nahmen, im Durchschnitt erst nach 25 Monaten eine Diagnose. Es konnte jedoch kein Zusammenhang zwischen soziodemografischen Faktoren der MmD oder deren Angehörigen und der Dauer bis zur Kontaktaufnahme zur Diagnoseabklärung belegt werden.

Auf Grundlage zweier niederländischer Kohortenstudien mit $M m D$ bei frühem Beginn und $M m D$ mit späten Beginn stellten van Vliet et al. [14] fest, dass im Vergleich zur Referenz Alzheimer-Demenz, Frontotemporale Demenz signifikant mit einer längeren Dauer bis zur Diagnose assoziiert ist. Vaskuläre Demenz wird hingegen signifikant früher erkannt. Die soziodemografischen Faktoren, 
Wohnsituation und Bildungsstand des MmD waren jedoch keine signifikanten Prädiktoren für die Dauer bis zur Diagnosestellung [14].

Cattel et al. [15] stratifizierten MmD, die in eine italienische Gedächtnisambulanz überwiesen wurden, auf Basis von bestehenden alltagspraktischen Fähigkeiten (ADLs). Bei Personen, die in der Lage waren ADLs selbstständig auszuführen, waren ein erhöhtes Alter, das weibliche Geschlecht sowie ein niedriger MMST-Wert signifikant mit einer längeren Dauer von den ersten Symptomen bis zur Überweisung zur Abklärung in die Gedächtnissprechstunde assoziiert. Im Gegensatz dazu zeigte sich, dass für Personen mit physischer Beeinträchtigung ein niedriger MMST-Wert mit einer kürzeren Zeitspanne bis zur Diagnose assoziiert ist [15].

\section{Ziel der Arbeit}

Im Rahmen der vorliegenden Analyse soll daher für den deutschen Versorgungskontext der Frage nach dem Zeitraum von den ersten subjektiv wahrgenommenen Symptomen durch die pflegenden Angehörigen bis zur formellen Diagnosestellung nachgegangen werden sowie potenzielle soziodemografische Prädiktoren für diese Zeitspanne identifiziert werden.

\section{Methodik}

\section{Studiendesign und Studienpopulation}

Die Betrachtung der Zeitdauer von den ersten wahrgenommen Symptomen bis zur Demenzdiagnose stellt einen Teilaspekt der multizentrischen Längsschnittstudie Bayerischer Demenz Survey (BayDem) dar, die in drei Regionen in Bayern durchgeführt wurde. Das übergeordnete Ziel von BayDem war es die spezifischen Bedarfe und die Versorgungssituation von MmD und deren pflegenden Angehörigen über den Erkrankungsverlauf hinweg zu erheben. Zum einen wurden die Entwicklung der medizinischen Parameter, der alltagspraktischen und kognitiven Fähigkeiten sowie die Lebensqualität der MmD erfasst. Zum anderen wurden die Bedürfnisse sowie die subjektive Belastung der pflegenden Angehörigen und die Inanspruchnahme von Gesundheitsdienstleistungen ermittelt [16]. Die 3 Studienregionen Dachau, Erlangen und Kronach stellen verschiedene demografische und sozioökonomische Gebiete mit unterschiedlichen Bevölkerungsentwicklungen dar. Durch Einbeziehung der Städte und Landkreise wurden sowohl urbane als auch ländliche Räume abgebildet. Teilnehmer/innen waren MmD (definiert nach ICD-10, F00-F03), die von ihren pflegenden Angehörigen zuhause versorgt wurden, wie auch die Angehörigen selbst. Um eine valide Abbildung der Versorgungsrealität zu gewährleisten, handelte es sich bei den Angehörigen um die Hauptpflegeperson bzw. Hauptunterstützer/in im unmittelbaren Lebensumfeld des MmD (z. B. Lebenspartner/in, Kinder, Schwiegerkinder oder enge Freunde).

\section{Rekrutierung und Follow-up}

Um den unterschiedlichen Zugangswegen von MmD Rechnung zu tragen, erfolgte die Rekrutierung der Teilnehmer/innen über vielfältige Einrichtungen (Beratungsstellen, niedergelassene Ärzte und Therapeuten, medizinische Versorgungszentren, Gedächtnisambulanzen, Pflegedienste, ehrenamtliche Dienste und Krankenhäuser). Hierfür wurden die lokalen Akteure identifiziert und konzep- tionell sowie organisatorisch eingebunden. Die Datenerhebung erfolgte durch geschulte Befrager/innen in Form von standardisierten persönlichen Interviews im häuslichen Umfeld. Die Daten wurden gemäß den internationalen Standards nach ICHOM bei Studieneinschluss (t0), nach 6 Monaten (t6) sowie nach 12 Monaten (t12) erhoben [17]. Als Basis für die beschriebene Fragestellung dienten Daten, die zum Erhebungszeitpunkt t0 erfasst wurden.

\section{Datenschutz}

Die MmD und deren Angehörige wurden vor Studieneinschluss umfassend aufgeklärt. Schriftliche Einverständniserklärungen wurden von den Teilnehmer/innen oder deren gesetzlichen Vertreter/innen eingeholt.

\section{Ethikvotum}

Vor Beginn der Studie wurde das Votum der Ethikkommission der Medizinischen Fakultät der Friedrich-Alexander-Universität Erlangen-Nürnberg eingeholt (Zeichen: 141_12B).

\section{Datenerhebung}

Es wurden soziodemografische Daten der MmD und der pflegenden Angehörigen erhoben. Zur Erhebung valider Diagnosen wurden im Rahmen der persönlichen Interviews die vorliegenden medizinischen Dokumente berücksichtigt. Da gemäß ICD-10-Definition eine Demenzdiagnose das Bestehen der Symptome über 6 Monate voraussetzt, wurde die Annahme getroffen, dass eine zeitgerechte Diagnosestellung innerhalb von 9 Monaten nach der Wahrnehmung der ersten Demenzsymptome erfolgen sollte. Die Zuordnung des Wohnsitzes der MmD zum ländlichen oder städtischen Raum beruht auf der Klassifikation des Bundesinstitutes für Bau-, Stadt- und Raumforschung.

\section{Statistische Analysen}

Binär-logistische Regressionen wurden für die Analyse der soziodemografischen Einflussfaktoren einer zeitgerechten Diagnosestellung durchgeführt. Für die Analysen wurde ein Signifikanzniveau von $5 \%$ angesetzt $(p<0,05)$.

Die Daten wurden mittels SPSS Software, Version 21 (International Business Machines Corporation (IBM), Armonk, New York, USA) analysiert und ausgewertet.

Die Ergebnisse der statistischen Analyse wurden in Anlehnung an die REST Guidelines dargestellt [18].

\section{Ergebnisse}

Retrospektive Daten zum Verlauf des Patientenpfades vom Zeitpunkt der ersten wahrgenommenen Symptome bis zur Erstdiagnose wurden von 276 Teilnehmerpaaren (MmD und pflegender Angehöriger) erfasst. Davon wurden 67,4\% $(n=186)$ in Akut- und Rehakliniken sowie angeschlossenen Gedächtnisambulanzen rekrutiert. Weitere 41 Teilnehmerpaare wurden von niedergelassenen Haus- und Fachärzten/innen (14,9\%), sowie 21 von ambulanten Pflegediensten $(7,6 \%)$ in die Studie aufgenommen. Beratungsstellen schlossen 19 $(6,9 \%)$ und sonstige Gesundheitsdienstleister 9 MmD-AngehörigerDyaden (3,3\%) ein.

Die soziodemografischen Merkmale der Teilnehmenden sind in - Tab. 1, 2 dargestellt. Der Anteil an männlichen teilnehmenden 
- Tab. 1 Charakteristika der teilnehmenden MmD.

\begin{tabular}{|c|c|c|c|}
\hline Merkmale & $\begin{array}{l}\text { Zeitgerechte } \\
\text { Diagnose } \\
(\leq 9 \text { Monate }) \\
(n=102)\end{array}$ & $\begin{array}{l}\text { Verspätete } \\
\text { Diagnose } \\
\text { (> } 9 \text { Monate) } \\
\text { ( } n=174)\end{array}$ & $\begin{array}{l}\text { Gesamt } \\
(n=276)\end{array}$ \\
\hline $\begin{array}{l}\text { Alter zum Zeitpunkt der } \\
\text { ersten wahrgenommen } \\
\text { Symptome } \\
\text { (in Jahren) } \\
\end{array}$ & $78,2 \pm 7,9$ & $73,7 \pm 9,4$ & $75,41 \pm 9,2$ \\
\hline \multicolumn{4}{|l|}{ Geschlecht } \\
\hline Weiblich, n (\%) & $47(46,1)$ & $94(54,0)$ & $141(51,1)$ \\
\hline Männlich, n (\%) & $55(53,9)$ & $80(46,0)$ & $135(48,9)$ \\
\hline \multicolumn{4}{|l|}{ Familienstand } \\
\hline Ledig, n (\%) & $1(1,0)$ & $4(2,3)$ & $5(1,8)$ \\
\hline Ehe/ Partnerschaft, n (\%) & $58(56,8)$ & $116(67,0)$ & $174(63,3)$ \\
\hline $\begin{array}{l}\text { Getrennt lebend/ } \\
\text { geschieden, n (\%) }\end{array}$ & $2(2,0)$ & $6(3,5)$ & $8(2,9)$ \\
\hline Verwitwet, n (\%) & $41(40,2)$ & $47(27,2)$ & $88(32)$ \\
\hline Keine Angabe, $n$ & - & 1 & \\
\hline \multicolumn{4}{|l|}{ Wohnsituation } \\
\hline Alleinlebend, n (\%) & $25(24,5)$ & $34(19,5)$ & $59(21,4)$ \\
\hline Nicht alleinlebend, n (\%) & $77(75,5)$ & $140(80,5)$ & $217(78,6)$ \\
\hline $\begin{array}{l}\text { Schulbildung in Jahren } \\
(\mathrm{MW} \pm \mathrm{SD})\end{array}$ & $8,7 \pm 1,3$ & $9,3 \pm 1,7$ & $9,1 \pm 1,6$ \\
\hline \multicolumn{4}{|l|}{ Wohnort } \\
\hline Ländlich, n (\%) & $35(34,3)$ & $50(28,7)$ & $85(30,8)$ \\
\hline Städtisch, n (\%) & $67(65,7)$ & $124(71,3)$ & $191(69,2)$ \\
\hline
\end{tabular}

- Tab. 2 Charakteristika der teilnehmenden pflegenden Angehörigen.

\begin{tabular}{|c|c|c|c|}
\hline Merkmale & $\begin{array}{l}\text { Zeitgerechte } \\
\text { Diagnose } \\
\text { ( } \leq 9 \text { Monate) } \\
\text { ( } n=102)\end{array}$ & $\begin{array}{l}\text { Verspätete } \\
\text { Diagnose } \\
\text { (> 9 Monate) } \\
(n=174)\end{array}$ & $\begin{array}{l}\text { Gesamt } \\
(n=276)\end{array}$ \\
\hline $\begin{array}{l}\text { Alter zum Zeitpunkt der } \\
\text { ersten wahrgenommen } \\
\text { Symptome (in Jahren) }\end{array}$ & $60,4 \pm 13,7$ & $57,8 \pm 12,8$ & $58,8 \pm 13,2$ \\
\hline \multicolumn{4}{|l|}{ Geschlecht } \\
\hline Weiblich, n (\%) & $75(73,5)$ & $124(71,3)$ & $199(72,1)$ \\
\hline Männlich, n (\%) & $27(26,5)$ & $50(28,7)$ & $77(27,9)$ \\
\hline \multicolumn{4}{|l|}{ Stellung zum MmD } \\
\hline (Ehe)-Partner/in, n (\%) & $46(45,1)$ & $90(51,7)$ & $136(49,3)$ \\
\hline Kind/Schwiegerkind, n (\%) & $51(50)$ & $74(42,5)$ & $125(45,3)$ \\
\hline Verwandte, n (\%) & $5(4,9)$ & $9(5,2)$ & $5,1(14)$ \\
\hline Freund/in, n (\%) & - & $1(0,6)$ & $0,4(1)$ \\
\hline
\end{tabular}

MmD (48,9\%) war nur sehr geringfügig niedriger als der Anteil der weiblichen teilnehmenden MmD. Etwa ein Fünftel der teilnehmenden MmD war alleinlebend (21,4\%). Bei den befragten Angehörigen handelt es sich überwiegend um (Ehe-)/Lebenspartner/innen der MmD (49,3\%) sowie Kinder bzw. Schwiegerkinder (45,3\%).

Aus Sicht der teilnehmenden pflegenden Angehörigen $(n=276)$ betrug die mediane Dauer von der Wahrnehmung der ersten Demenzsymptome bis zur Diagnosestellung 16,0 Monate (Min.: 0 Monate; Max.: 139 Monate; MW: 23,7 Monate; SD: 25,7 Monate). 37\% $(n=102)$ der Teilnehmenden erhielten innerhalb der ersten 9 Monate nach Identifikation erster Symptome die Erstdiagnose.
Insgesamt wurde die erste Kontaktaufnahme zur Diagnoseabklärung vorwiegend von den Kindern des $\operatorname{MmD}(37,1 \% ; n=99)$ sowie den (Ehe-)Partnern/innen (33,0\%; $n=88$ ) initiiert. 29,6\%; ( $n=79)$ der Teilnehmenden gaben an, dass ärztliches bzw. pflegerisches Personal eine erste Diagnoseabklärung veranlassten. Nur knapp jeder zehnte teilnehmende $\mathrm{MmD}(9,7 \% ; n=26)$ war zudem initial an der ersten Kontaktaufnahme zum Zwecke der Abklärung der Diagnose beteiligt.

Als Ergebnis der in > Tab. 3 dargestellten binär-logistischen Regressionsanalyse, konnten 3 signifikante Einflussfaktoren identifiziert werden.

Es zeigte sich, dass die Wahrscheinlichkeit einer Diagnose innerhalb von 9 Monaten signifikant sinkt, je mehr Schuljahre die MmD absolviert hatten (OR: 0,787).

Zudem steigt die Chance einer Diagnose innerhalb von 9 Monaten mit zunehmendem Alter der MmD signifikant an. Die Ausprägung dieses Effektes erscheint jedoch mit einem Anstieg der Chance um 1,061 pro Lebensjahr als äußerst gering. Wird beispielhaft eine 5 Jahres Skalierung des Alters zugrunde gelegt, wird deutlich, dass sich die Chance einer zeitgerechten Diagnose zwischen Personen mit einer Altersdifferenz von 5 Jahren, um das 1,343-Fache erhöht.

Des Weiteren ist die Chance einer Diagnose innerhalb von 9 Monaten für weibliche MmD im Vergleich zu männlichen signifikant verringert (OR: 0,503). Eine signifikante Assoziation zwischen dem Wohnort (ländliche vs. städtische Regionen) oder der Wohnsituation der MmD (alleinlebend oder nicht alleinlebend) und einer Diagnose innerhalb von 9 Monaten nach der Erkennung erster Demenzsymptome wurde nicht beobachtet.

Mit einer Varianzaufklärung von 13,4\% (Nagelkerke R²), ist jedoch davon auszugehen, dass eine zeitgerechte Diagnosestellung noch durch weitere Einflussfaktoren determiniert wird. In den nach Geschlecht stratifizierten binären Regressionsanalysen in > Tab. 4 wird deutlich, dass nur für die männlichen MmD eine signifikante Assoziation zwischen einer zeitgerechten Diagnosestellung und dem Alter des MmD zum Zeitpunkt der ersten wahrgenommen Symptome (OR: 1,091) sowie der Schulbildung der MmD (OR: 0,755 ) besteht (Nagelkerke $R^{2}=0,217$ ).

\section{Diskussion}

Die vorliegende Studie zeichnet sich durch ein multizentrisches Design aus, das sowohl unterschiedliche geografische Regionen als auch eine Vielzahl verschiedener Rekrutierungsquellen beinhaltet. Die drei Regionen charakterisieren sich dabei durch unterschiedliche demografische, sozioökonomische und geografische Gegebenheiten. Zudem schließt die Studie sowohl Menschen im städtischen, als auch im ländlichen Bereich ein. Das Studiendesign diente dazu, eine möglichst hohe Validität der Daten sicherzustellen. Durch die Durchführung der Interviews im häuslichen Umfeld der Teilnehmer konnte zudem ein realistisches Bild der Lebenssituation gewonnen werden.

Die ermittelte durchschnittliche Dauer von etwa 2 Jahren von der Erkennung erster Demenzsymptome durch die Angehörigen bis zur Erstdiagnose einer Demenz, fällt im Vergleich zu bisherigen internationalen Befunden [11-14] geringer aus. Gründe für diese länderspezifischen Unterschiede könnten zum einen im unter- 
- Tab. 3 Assoziationen zwischen einer zeitgerechten Diagnosestellung 9 Monate nach den ersten wahrgenommenen Symptomen und soziodemografischen Merkmalen der MmD.

\begin{tabular}{|l|l|l|l|l|l|l|l|l|}
\hline & $\begin{array}{l}\text { Regressions- } \\
\text { koeffizient } \boldsymbol{\beta}\end{array}$ & $\begin{array}{l}\text { Standard- } \\
\text { fehler }\end{array}$ & $\begin{array}{l}\text { Waldkoeffi- } \\
\text { zient }\end{array}$ & df & p-Wert & OR & 95,0\% Konfidenzintervall für OR \\
\hline & & & & & & & Unterer Wert & Oberer Wert \\
\hline $\begin{array}{l}\text { Alter zum Zeitpunkt der ersten } \\
\text { wahrgenommen Symptome }\end{array}$ & 0,059 & 0,017 & 12,444 & 1 & $<0,001$ & 1,061 & 1,027 \\
\hline Schulbildung in Jahren & $-0,240$ & 0,099 & 5,902 & 1 & 0,015 & 0,787 & 0,648 & 0,955 \\
\hline Geschlecht (Ref.: männlich) & $-0,688$ & 0,282 & 5,948 & 1 & 0,015 & 0,503 & 0,289 \\
\hline Wohnsituation (Ref.: nicht alleinlebend) & 0,160 & 0,331 & 0,235 & 1 & 0,628 & 1,174 & 0,614 & 0,874 \\
\hline Wohnort (Ref.: ländlicher Raum) & $-0,275$ & 0,292 & 0,887 & 1 & 0,346 & 0,760 & 0,429 \\
\hline Nagelkerke R2=0,134 & & & & \\
\hline $\begin{array}{l}\text { df=Freiheitsgrade; OR= Odds ratio; Kodierung: Abhängige Variable zeitgerechte Diagnosestellung innerhalb von 9 Monaten (1), verspätete Diagnosestellung } \\
\text { nach 9 Monaten (0); Geschlecht: weiblich (1), männlich (0); Wohnsituation: alleinlebend (1), nicht alleinlebend (0); Wohnort: Städtisch (1), ländlich (0) }\end{array}$ \\
\hline
\end{tabular}

- Tab. 4 Assoziationen zwischen einer zeitgerechten Diagnosestellung 9 Monate nach den ersten wahrgenommenen Symptomen und soziodemografischen Merkmalen der MmD stratifiziert nach Geschlecht.

\begin{tabular}{|c|c|c|c|c|c|c|c|c|}
\hline & \multicolumn{4}{|c|}{ Männlich } & \multicolumn{4}{|c|}{ Weiblich } \\
\hline & $\begin{array}{l}\text { Regressions- } \\
\text { koeffizient } \beta\end{array}$ & SE & p-Wert & OR & $\begin{array}{l}\text { Regressions- } \\
\text { koeffizient } \beta\end{array}$ & SE & p-Wert & OR \\
\hline $\begin{array}{l}\text { Alter zum Zeitpunkt der ersten wahrge- } \\
\text { nommen Symptome }\end{array}$ & 0,088 & 0,025 & $<0,001$ & 1,091 & 0,039 & 0,024 & 0,104 & 1,039 \\
\hline Schulbildung in Jahren & $-0,282$ & 0,119 & 0,018 & 0,755 & $-0,122$ & 0,183 & 0,504 & 0,885 \\
\hline Wohnsituation (Ref.: nicht alleinlebend) & $-0,726$ & 0,620 & 0,242 & 0,484 & 0,578 & 0,400 & 0,149 & 1,782 \\
\hline Wohnort (Ref.: ländlicher Raum) & $-0,272$ & 0,443 & 0,540 & 0,762 & $-0,327$ & 0,398 & 0,412 & 0,721 \\
\hline Nagelkerke $\mathrm{R}^{2}=$ & 0,217 & & & & 0,080 & & & \\
\hline
\end{tabular}

schiedlichen öffentlichen Bewusstsein und der Sensibilität für Demenzen in der Bevölkerung sowie der medizinischen und pflegerischen Leistungserbringer vermutet werden [12]. Möglicherweise ist die beobachtete Differenz auch z. T. in der Bandbreite der einbezogenen Rekrutierungsorte, die auch Akut- und Rehakliniken umfassten, begründet.

Obwohl eine zeitgerechte Diagnosestellung für MmD und deren unterstützenden Angehörigen die Basis für eine optimale Versorgungsplanung und Inanspruchnahme von Unterstützungsleistungen bildet [7, 19], wurde es bisher wenig erforscht, welchen Einfluss soziodemografische Faktoren auf die Zeitspanne von der Erkennung der ersten Symptome bis zur Diagnosestellung besitzen.

Die vorliegende Analyse zeigt eine signifikant positive, jedoch äußerst schwache Assoziation zwischen steigendem Alter von männlichen MmD und einer Diagnose innerhalb von 9 Monaten nach Erkennung erster Symptome durch die Angehörigen. Untersuchungen mit MmD im mittleren Lebensalter (early-onset dementia) zeigen, dass die Zeitspanne bis zur Diagnosestellung für diese Personengruppe, die einen Symptombeginn vor dem 65. Lebensjahr aufweisen, im Durchschnitt höher ausfällt als bei MmD mit späten Beginn [14, 20]. Gründe für diese Differenz liegen unter anderem darin begründet, dass die meisten Angehörigen von MmD im mittleren Lebensalter auftretende Symptome nicht mit einer Demenz assoziieren und auch vermehrt initiale Fehldiagnosen berichtet wurden [21].
Im Einklang mit den dargestellten Ergebnissen, wurde auch in einer weiteren Studie [15] berichtet, dass weibliche MmD eine signifikant erhöhte Wahrscheinlichkeit für eine verspätete Diagnosestellung besitzen. Die bestehende Evidenz zu geschlechtsspezifischen Unterschieden im Hilfesuchverhalten im Bereich von psychischen Erkrankungen belegt hingegen, dass es wahrscheinlicher ist, dass weibliche Personen mit psychischen Belastungen Unterstützung aufsuchen [22]. Es wurde jedoch auch nachgewiesen, dass die Erkennung von Gedächtnisstörungen bei MmD durch Angehörige mit zunehmenden Verhaltensauffälligkeiten assoziiert ist [23]. Studien die Geschlechterunterschiede bei neuropsychiatrischen Symptomen von MmD untersuchten ergaben, dass Frauen mit Alzheimer-Demenz häufiger Depressionen und Ängste zeigten als Männer [24, 25]. Möglicherweise könnte es daher auch hier seitens der Angehörigen zu Fehlattributionen von ersten Symptomen kommen.

Die vorgestellten Ergebnisse zeigen, dass die Chance einer Diagnose innerhalb von 9 Monaten signifikant mit der Anzahl der Schuljahre die die MmD absolviert hatten, sinkt. Eine Metaanalyse belegt, dass ein niedriger Bildungsgrad im Zusammenhang mit einem erhöhten Demenzrisiko steht. Ebenso wurde ermittelt, dass sich MmD mit einer Hochschulausbildung zum Zeitpunkt der ersten Vorstellung in einem früheren Demenzstadium befinden und höhere Testergebnisse vorwiesen. Es gibt Hinweise darauf, dass der Verlust der kognitiven Funktionen bei Menschen mit höherer Bildung bis zu einem gewissen Zeitpunkt langsamer verläuft, wobei 
anschließend ein schnellerer Abbau der kognitiven Funktionen zu beobachten ist [26].

Übereinstimmend mit einer vergleichbaren Studie [14] ist bei der Interpretation der vorliegenden Ergebnisse zu beachten, dass der Anteil der aufgeklärten Varianz relativ gering ausfiel und demzufolge noch weitere wesentliche unberücksichtigte Variablen eine zeitgerechte Diagnose beeinflussen. Es kann daher nicht ausgeschlossen werden, dass dies zu einer potenziellen Verzerrung der Schätzer führen könnte.

Eine aktuelle Befragung von Wangler et al. [27] in RheinlandPfalz verdeutlicht bspw., dass ein beträchtlicher Anteil der befragten Hausärzte/innen (42,1\%) den Wert einer frühzeitigen Demenzdiagnostik auf Grund von einem wahrgenommenen Mangel an therapeutischen Handlungsoptionen anzweifelt. Bei Hausärzten/innen mit geriatrischer Weiterbildung war der diagnostische und therapeutische Nihilismus seltener zu beobachten (35,0\%). Zudem wurden Screeninginstrumente deutlich häufiger von Hausärzten/innen mit Zusatz-Weiterbildung Geriatrie angewendet. Stigmatisierung und ein weitverbreiteter therapeutischer Nihilismus in der primärärztlichen Versorgung wurden auch im Rahmen einer Fokusgruppenanalyse von Demenzexperten aus 8 europäischen Ländern als zentrale Hindernisse für eine zeitgerechte Demenzerkennung und Diagnostik identifiziert [28].

Ferner könnte eine erhöhte Sensibilisierung des öffentlichen Bewusstseins für die Bandbreite von Demenzsymptomen zu einer zeitgerechten Diagnosestellung beitragen. In der Literatur konnte belegt werden, dass die Zuordnung der Symptome durch die jeweiligen Angehörigen als alters- oder persönlichkeitsbedingt, die Suche nach Unterstützung signifikant verzögerte. Angehörige die anhand der wahrgenommen Symptome hingegen eine Demenz vermuteten, besaßen eine fast 6 -fach höhere Chance innerhalb von 3 Monaten Kontakt zu Gesundheitsanbietern zur Symptomabklärung aufzunehmen [13].

Ein systematisches Review zeigt, dass international bisher nur wenig Evidenz im Bereich von Interventionen zur Steigerung der Diagnoserate sowie einer zeitgerechteren Diagnosestellung besteht. Es konnte belegt werden, dass Schulungen für Hausärzte/ innen die Rate der Verdachtsfälle steigern, jedoch keinen Einfluss auf die Diagnoserate oder den Zeitraum bis zur Diagnosestellung besitzen. Zudem gibt es Hinweise darauf, dass der Zugang zu spezialisierten Memory-Kliniken zu einer zeitgerechteren Diagnosestellung beitragen kann [29].

In einem Scoping Review von Barth et al. [30] zeigte sich, dass die Barrieren für eine zeitgerechte Diagnose insbesondere im ländlichen Raum hoch sind. Gleichwohl konnte festgestellt werden, dass Telehealth-Anwendungen, wie Videokonferenzen, ein geeignetes Mittel für eine Demenzdiagnose darstellen können. Zudem wurde deutlich, dass Interventionen für Hausärzt/innen (Online-Unterstützungstools, Schulungen und Trainings) zu einer Verbesserung des Wissens und Selbstvertrauens bezüglich der Diagnosestellung führen [30]. Eine deutsche Studie konnte darüber hinaus zeigen, dass ein systematisches Screening älterer Patienten hinsichtlich des Abbaus der kognitiven Leistungsfähigkeit im Rahmen der Routineversorgung zu einer Steigerung der Diagnoserate führt [3]. Auf Basis der Befragung von Hausärzten/innen in Rheinland-Pfalz wur- den neben der Erhöhung des Bewusstseins für die Bedeutung einer zeitgerechten Diagnosestellung im Sinne der Sicherstellung einer gelingenden Versorgung auch eine verstärkte Einbindung des Praxispersonals als Ansatzpunkte für die Optimierung der hausärztlichen Demenzdiagnostik identifiziert [27].

\section{Limitationen}

Die Rekrutierung der MmD fand über Beratungsstellen, niedergelassene Ärzte/innen und Therapeut/innen, medizinische Versorgungszentren, Gedächtnisambulanzen, Pflegedienste, ehrenamtliche Dienste und Krankenhäuser statt, mit dem Ziel, möglichst aussagekräftige Daten zu gewinnen. Nichtsdestotrotz kann eine Selektion von Teilnehmer/innen mit spezifischen Versorgungshintergründen nicht ausgeschlossen werden.

Zudem wurden stets Dyaden von MmD und deren Angehörigen in die BayDem Studie aufgenommen. Es besteht daher die Möglichkeit eines potenziellen Selektion Bias, da für das Demenzsyndrom sensibilisierte und informierte pflegende Angehörige eher zur Teilnahme eingewilligt haben könnten, als Angehörige die eine Demenzdiagnose negieren.

Die Angaben der pflegenden Angehörigen zur Wahrnehmung der ersten Symptome beruhen auf subjektiven Erinnerungen. Als schwierig gestaltet sich hierbei, dass den neuauftretenden Verhaltensauffälligkeiten oder Gedächtniseinschränkungen von den Angehörigen kein unmittelbarer Krankheitswert beigemessen wird und deshalb keine objektivierbaren Aufzeichnungen verfügbar sind. Deshalb ist ein Verzerrungsrisiko aufgrund eines Erinnerungsbias nicht gänzlich auszuschließen.

\section{Schlussfolgerungen}

Es zeigte sich, dass die Diagnosestellung häufig mit deutlicher Verzögerung erfolgt. Deshalb erscheint es notwendig, dass im primärärztlichen Bereich strukturierte Zugangswege zu einer „zeitgerechten Diagnostik“ weiterentwickelt werden. Sogenannte „zugehende" Beratungs- und Diagnostikangebote sollten deshalb zukünftig verstärkt ausgebaut werden.

\section{Förderung}

Das Projekt BayDem wurde durch das Bayerische Staatsministerium für Gesundheit und Pflege (StMGP) gefördert (Förderkennzeichen: G42b-G8092.9-2014/10-7).

\section{Danksagung}

Die vorliegende Arbeit wurde von Franziska Wolff als Teil der Anforderungen zur Erlangung des Grades „Dr. rer. biol. hum.“ an der Medizinischen Fakultät der Friedrich-Alexander-Universität Erlangen-Nürnberg (FAU) erstellt.

\section{Interessenkonflikt}

Die Autorinnen/Autoren geben an, dass kein Interessenkonflikt besteht. 
[1] Bickel H. Die Häufigkeit von Demenzerkrankungen (06.2018). Im Internet: https://www.deutsche-alzheimer.de/fileadmin/alz/pdf/ factsheets/infoblatt1_haeufigkeit_demenzerkrankungen_dalzg.pdf; Stand: 07.05.2019

[2] Prince M, Bryce R, Ferri C. World Alzheimer Report 2011. The benefits of early diagnosis and intervention (09.2011). Im Internet: https:// www.alz.co.uk/research/WorldAlzheimerReport2011.pdf; Stand: 12.06.2017

[3] Eichler T, Thyrian JR, Hertel J et al. Rates of formal diagnosis in people screened positive for dementia in primary care: results of the DelpHi-Trial. J Alzheimers Dis 2014; 42: 451-458

[4] Pentzek M, Fuchs A, Wiese B et al. General practitioners' judgment of their elderly patients' cognitive status. J Gen Intern Med 2009; 24: 1314-1317

[5] Pentzek M, Wollny A, Wiese B et al. Apart from nihilism and stigma: what influences general practitioners' accuracy in identifying incident dementia? Am J Geriatr Psychiatry 2009; 17: 965-975

[6] Bradford A, Kunik ME, Schulz P et al. Missed and delayed diagnosis of dementia in primary care: prevalence and contributing factors. Alzheimer Dis Assoc Disord 2009; 23: 306-314

[7] Dubois B, Padovani A, Scheltens P et al. Timely Diagnosis for Alzheimer's Disease: A Literature Review on Benefits and Challenges. Journal of Alzheimer's Disease 2015; 49: 617-631

[8] Brooker D, Fontaine JL, Evans $S$ et al. Public health guidance to facilitate timely diagnosis of dementia: ALzheimer's COoperative Valuation in Europe recommendations. International Journal of Geriatric Psychiatry 2014; 29: 682-693

[9] van den Dungen P, van Kuijk L, van Marwijk $\mathrm{H}$ et al. Preferences regarding disclosure of a diagnosis of dementia: a systematic review. Int Psychogeriatr 2014; 26: 1603-1618

[10] Luck T, Luppa M, Sieber J et al. Attitudes of the German General Population toward Early Diagnosis of Dementia - Results of a Representative Telephone Survey. PLoS ONE 2012; 7: e50792

[11] Speechly CM, Bridges-Webb C, Passmore E. The pathway to dementia diagnosis. Med J Aust 2008; 189: 487-489

[12] Chrisp TAC, Thomas BD, Goddard WA et al. Dementia timeline: Journeys, delays and decisions on the pathway to an early diagnosis. Dementia 2011; 10: 555-570

[13] Feldman L, Wilcock J, Thune-Boyle I et al. Explaining the effects of symptom attribution by carers on help-seeking for individuals living with dementia. Dementia (London) 2015; 16: 375-387

[14] van Vliet D, de Vugt ME, Bakker $C$ et al. Time to diagnosis in young-onset dementia as compared with late-onset dementia. Psychol Med 2012; 43: 423-432

[15] Cattel C, Gambassi G, Sgadari A et al. Correlates of delayed referral for the diagnosis of dementia in an outpatient population. J Gerontol A Biol Sci Med Sci 2000; 55: M98-M102
[16] Schaller S, Marinova-Schmidt V, Setzer M et al. Bayerischer Demenz Survey: Methodik einer Längsschnitterhebung zur Versorgungssituation bei Demenz. Das Gesundheitswesen 2015; 77

[17] ICHOM (International Consortium for Health Outcomes Measurement) Dementia. Data Collection Reference Guide (03.04.2017). Im Internet: https://ichom.org/files/medical-conditions/dementia/dementia-reference-guide.pdf; Stand: 30.11 .2018

[18] Launay E, Cohen JF, Bossuyt PM et al. Reporting studies on time to diagnosis: proposal of a guideline by an international panel (REST). BMC Medicine 2016; 14: 146

[19] De Vugt ME, Verhey FR. The impact of early dementia diagnosis and intervention on informal caregivers. Prog Neurobiol 2013; 110: 21

[20] Draper B, Cations M, White F et al. Time to diagnosis in young-onset dementia and its determinants: the INSPIRED study. Int J Geriatr Psychiatry 2016; 31: 1217-1224

[21] van Vliet D, de Vugt ME, Bakker C et al. Caregivers' perspectives on the pre-diagnostic period in early onset dementia: a long and winding road. Int Psychogeriatr 2011; 23: 1393-1404

[22] Tedstone Doherty D, Kartalova-O'Doherty Y. Gender and self-reported mental health problems: predictors of help-seeking from a general practitioner. Br J Health Psychol 2010; 15: 213-228

[23] Eustace A, Bruce I, Coen R et al. Behavioural disturbance triggers recognition of dementia by family informants. Int J Geriatr Psychiatry 2007; 22: 574-579

[24] Spalletta G, Musicco M, Padovani A. Neuropsychiatric symptoms and syndromes in a large cohort of newly diagnosed, untreated patients with Alzheimer disease. Am J Geriatr Psychiatry 2010; 18: 1026-1035

[25] Lee J, Lee KJ, Kim H. Gender differences in behavioral and psychological symptoms of patients with Alzheimer's disease. Asian J Psychiatr 2017; 26: 124-128

[26] Meng X, D’Arcy C. Education and Dementia in the Context of the Cognitive Reserve Hypothesis: A Systematic Review with Meta-Analyses and Qualitative Analyses. PLoS One 2012; 7: e38268

[27] Wangler ], Fellgiebel A, Jansky M. Hausärztliche Demenzdiagnostik Einstellungen, Vorgehensweisen und Herausforderungen von Hausärzten in Rheinland-Pfalz. Dtsch med Wochenschr 2018; 143: e165-e171

[28] Vernooij-Dassen M], Moniz-Cook ED, Woods RT et al. Factors affecting timely recognition and diagnosis of dementia across Europe: from awareness to stigma. Int J Geriatr Psychiatry 2005; 20: 377-386

[29] Mukadam N, Cooper C, Kherani N et al. A systematic review of interventions to detect dementia or cognitive impairment. Int I Geriatr Psychiatry 2014; 30: 32-45

[30] Barth J, Nickel F, Kolominsky-Rabas PL. Diagnosis of cognitive decline and dementia in rural areas - A scoping review. Int J Geriatr Psychiatry 2018; $33:$ 459-47 